\title{
A Driver Assaulting Event Detection Using Intel Real-Sense Camera
}

\author{
Jae-Gon Yoo ${ }^{1}$, Dong-Kyun $\mathrm{Kim}^{2}$, Seung Joo Choi ${ }^{3}$, Handong $\mathrm{Lee}^{4}$ and Jong-Bae \\ $\operatorname{Kim}^{5^{*}}$ \\ 1,3,4,5* Graduate of Software, Soongsil University, Seoul, 156-743, Korea \\ ${ }^{2}$ Department of IT Policy and Management, Graduate School of Soongsil \\ University, Seoul, 156-743, Korea \\ 1'worhs0410@gmail.com,22dongkyun@gmail.com, ${ }^{3}$ csj5183@gmail.com, \\ ${ }^{4}$ hansleo8935@gmail.com, ${ }^{5 *}$ kjb123@ssu.ac.kr
}

\begin{abstract}
Biometrics refers to a technology that applies biological or behavioral characteristics such as fingerprint, face, iris, retina, vein, and voice, signature to security system. Since the 9/11 terror attack that occurred in the 9/11, all the countries in the world had been much interested in security using biometrics and the importance of protection and security of information has risen. Accordingly, the need for verifying identity and personal authentication has increased.

Advantage of face detection and recognition, one of above mentioned biometrics is that it is inexpensive in building new physical infrastructure. Unlike other biometrics such as fingerprint recognition and iris recognition, face detection is non touch mode. For this reason, face detection is applicable to such diverse fields such as finance, robot, and access control and surveillance camera. The disadvantage of image data is that it takes more time and costs more than data processing using sensor. Advance in relevant technology such as image recognition technology, communication infrastructure, bulk data processing environment led to high efficiency at low cost.

This study aims to verify identity of passengers of limited indoor area such as taxi and bus and track behavioral trajectory of relevant person by making good use of face detection, face recognition and object tracking technology. This study is to accumulate data that are applicable to recognition of crime pattern and the understanding of movement by detecting attack events or recording movement log.
\end{abstract}

Keywords: Detecting Driver Assaulting, Real-Sense Camera, Event Detection, Public Transportation Driver

\section{Introduction}

Biometrics refers to a technology that applies biological or behavioral characteristics such as fingerprint, face, iris, retina, vein, voice, and signature to security system. Since the 9/11 terror attack that occurred in the USAall the countries in the world had been much interested in security using biometrics and the importance of protection and security of information has risen. Accordingly, the need for verifying identity and personal authentication has increased.

The advantage of face detection and recognition, one of above mentioned biometrics is that it is inexpensive in building new physical infrastructure. Unlike other biometrics such as fingerprint recognition and iris recognition, face detection is non touch mode. For this reason, face detection is applicable to such diverse fields such as finance, robot, and

$5^{\star}$ Corresponding author. Tel. : +82-10-9027-3148.

Email address: kjb123@ssu.ac.kr(Jong-Bae Kim). 
access control and surveillance camera. The disadvantage of image data is that it takes more time and cost more than data processing using sensor. Advance in relevant technology such as image recognition technology, communication infrastructure, bulk data processing environment led to high efficiency at low cost.

Detecting face accurately from image acquired is required to apply face detection and recognition technology. Face detection means judging whether there is human face in given image and designating location and scope of face in image. Face detection includes Knowledge Based Method that uses prior knowledge on components of face, Feature Invariant Approach that extracts using structural characteristics of shape, Template Matching Method that extracts using correlation between structural pattern and image and Appearance-Based Method that extracts based on learning data. Face recognition checks whether there is face image in database. Face recognition includes one using statistics, one using neural network and one based on characteristics.

CCTV based security has studied object tracking and movement trajectory analysis actively. Police and public institutions recognize the importance of CCTV based security. Also, studies of of building intelligent image surveillance solution by predicting risks such as analysis of abnormal behavior pattern of object based on specific space has been conducted.

This study aims to verify the identity of passengers of limited indoor area such as taxi and bus and track behavioral trajectory of relevant person by making good use of face detection, face recognition and object tracking technology. This study aims to accumulate data that are applicable to recognition of crime pattern and the understanding of movement by detecting attack events or recording movement log [1].

\section{Related Works}

This study uses Face Detection and Recognition and Object Tracking using image processing as one of biometrics. Unlike biometrics including fingerprint recognition, face detection and recognition is more inexpensive in equipment than other methods. Face detection and recognition is non touch mode. Biometrics using visual data is required to process a large amount of visual data which takes much time to process data. Pretreatment is needed to reduce processing time. Filtering, binarization and edge detection are applicable to pretreatment [2].

\subsection{Face Detection}

As shown in Table 1, face detection is composed of various methods for example Knowledge Based Method that uses prior knowledge of components of human face, Feature Invariant Approach that detects by extracting structural characteristics of human shape which is irrelevant to a change in pose, observation time or light source, Template Matching Method that detects by entering face composition standard pattern and extracting correlation with input image and Appearance Based Method that detects by extracting face expression method from learning data[2][5]. Figure 1 shows a picture of relations between patterns of face template sections.

Table 1. Classification Table of Face Detection Methods

\begin{tabular}{|l|l|}
\hline Approach & Representative works \\
\hline Knowledge Based Method & Multi-Resolution Rule Based Method \\
\hline Feature Invariant Approaches & Grouping of Edges \\
$-\quad$ Facial Features & Space Gray-Level Dependence Matrix of Face \\
$-\quad$ Texture & Pattern \\
$-\quad$ Skin & Mixture of Gaussian \\
$-\quad$ Multiple Features & Integration of Skin Color, Size and Shape \\
\hline
\end{tabular}




\begin{tabular}{|c|l|}
\hline $\begin{array}{c}\text { Template Matching Method } \\
-\quad \text { Predefined Face Templates }\end{array}$ & $\begin{array}{l}\text { Shape Template } \\
\text { Active Shape Model }\end{array}$ \\
\hline$\quad$ Deformable Templates & Eigen Vector Decomposition and Clustering \\
Appearance Based Method & $\begin{array}{l}\text { Gaussian Distribution and Multilayer Perception } \\
\text { Ensemble of Neural Networks and Arbitration } \\
\text { Schemes } \\
-\quad \text { Eigen Face }\end{array}$ \\
$-\quad$ Neural Network & $\begin{array}{l}\text { Soint Statistics of Local Appearance and Position } \\
\text { Higher Order Statistics with HMM }\end{array}$ \\
$-\quad$ Support Vector Machine & Kullback Relative Information \\
$-\quad$ Naïve Bayed Classifier & Fast Approach with Adaboost \\
$-\quad$ Hidden Markov Model & \\
$-\quad$ Bnformation Theoretical Approach & \\
\hline
\end{tabular}

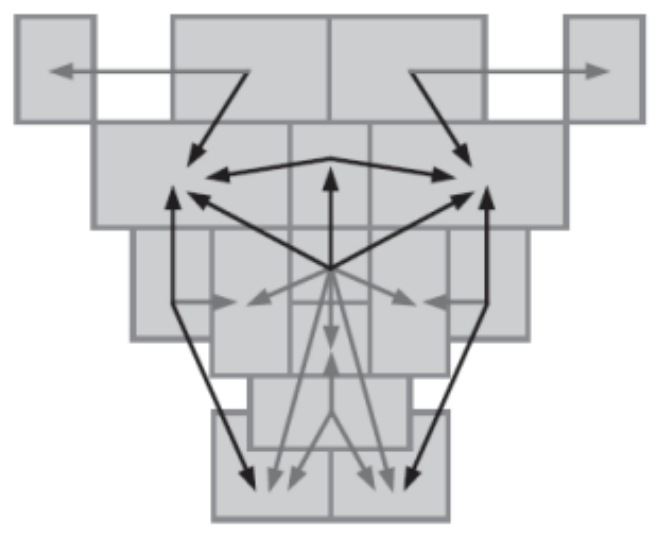

Figure 1. Example of Face Detection using Template Matching Method

\subsection{Face Recognition}

Face Recognition can be defined as a method that checks whether there are data that coincides in face database for a given image or image data. Further information such as skin color, age, gender can be used to limit the scope of database search. Such face recognition consists of separation of face from image, extraction of characteristics of face and figure classification process through search in database. Separation of face area which is required for face recognition was already mentioned in face detection.

Face is detected from input image and then extracted and aligned. Face alignment process is performed based on extracted face. This process is required to get data which is comparable to face database. Figure 2 below shows examples of above mentioned process.

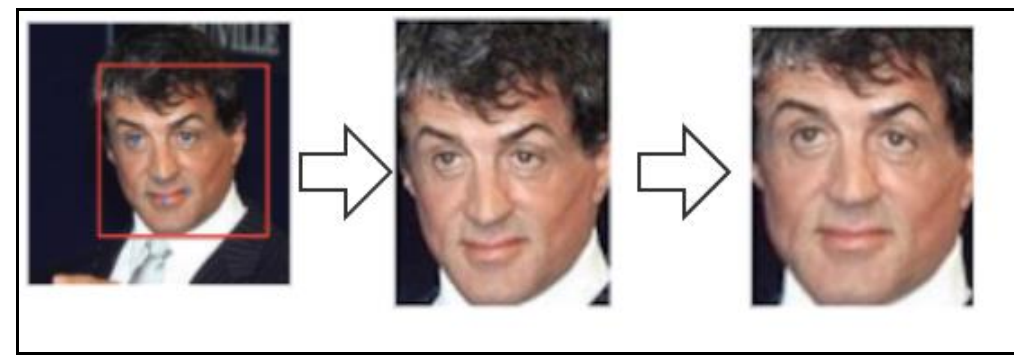

Figure 2. Preprocessing of Face Recognition 
Faces extracted from input image do not have typical shape because there is a difference in illumination and rotation of face. Face alignment is required to compensate for low recognition. Histogram Equalization, Histogram Stretching and Gausian Convolution are used to reduce effect of illumination. There are methods which creates new model instance in space reduced to lower level using PCA (Principal Component Analysis) and AAM (Active Appearance Model) as a method that compensates for rotation and size of face. As seen in Figure 1 the process can be identified as the process which compares the face on the database based on face extracted from input image. The face located in the middle shows that head is lowered. The picture on the right shows that the rotation angle of the face has been compensated through face alignment process [8].

Extraction of characteristics that can express human face from aligned face image is required. Extracted characteristics are used in recognizing the by comparing similarity through database on characteristics of face and sorter [6][7].

\subsection{Object Tracking}

Image processing is performed by using image data obtained from fixed camera in image based surveillance system and a change in extracted data is detected and detecting and tracking of an object are conducted based on it [3][4]. This study aims at finding movement area of humans on screen by taking humans as an object. Tracking has the following difficulties because humans are not fixed object and there are various methods in detecting and tracking humans; first, human movement is difficult to predict and shape changes rapidly depending on movement. Second, objects are overlapped in part or whole by human movement. Part or whole of a human is hidden or overlapped. Third, humans vary in size and shape and appearance of humans can vary easily depending on clothes, cap or glasses. Fourth, if a human moves rapidly, the observer is likely to lose sight of an object being tracked by blurring of human object in each frame. Fifth, noise such as shade occurring in light source causes difficulty in tracking [9].

Object tracking has a flow. Characteristics of an object are extracted from input image and an object is detected and interest area is divided. Then, object tracking is performed by applying object tracking algorism.

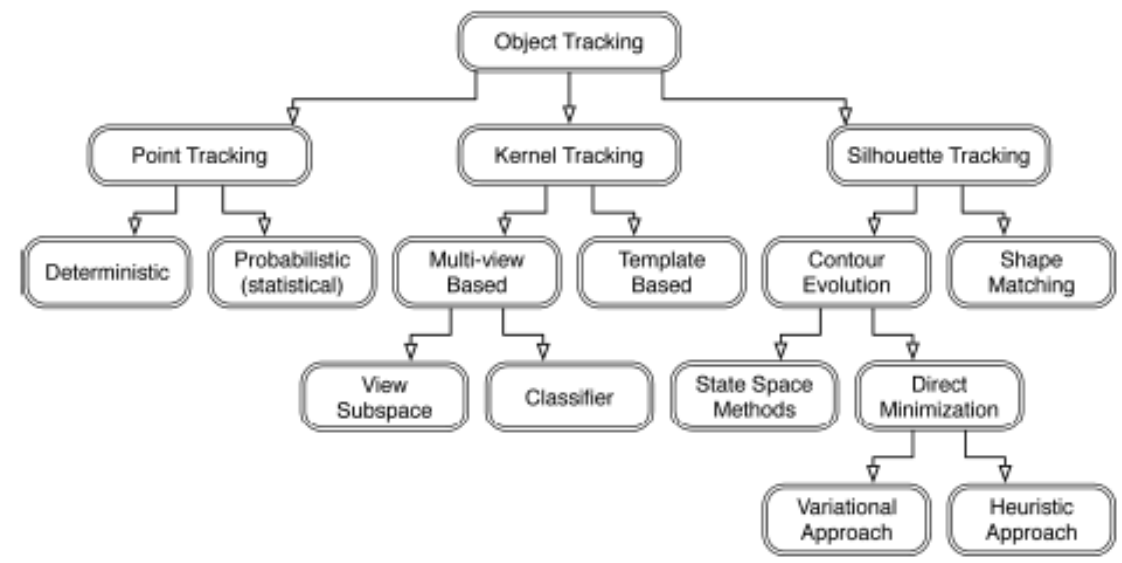

Figure 3. Diagram of Object Tracking Algorithm [3]

Figure 3 shows the diagram of object tracking algorithms. Object tracking can be divided into three categories with Point Tracking, Kernal Tracking and Silhouette Tracking. These also can be divided sub categories. Figure 4 shows characteristics of three categories[3][9]. 


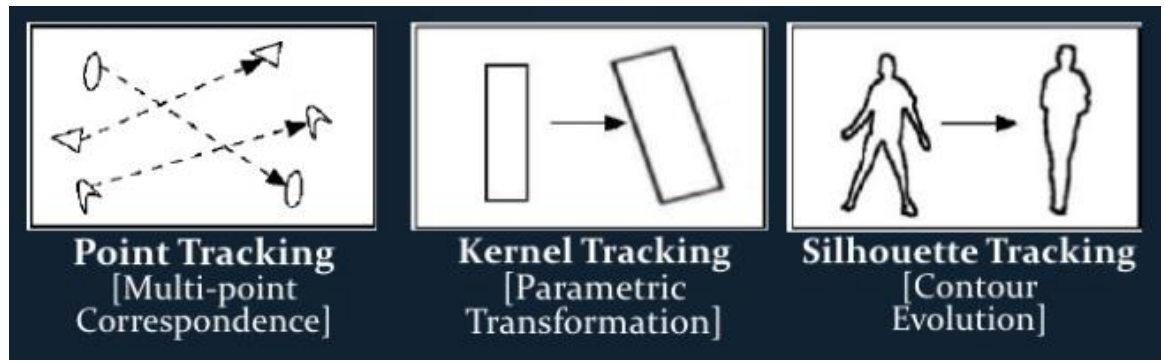

Figure 4. Characteristics of Point Tracking, Kernel Tracking and Silhouette Tracking

\section{Driver Assaulting Event Detection System}

For basic direction of this system, the scope of this study is limited to indoor area. This study is divided into detection-recognition and detection-tracking. Face is detected for a figure that enters a designated area in detection-recognition phase and whether there is a figure in specific figure database and the information is delivered to image processing system. If the figure exists in specific figure database, surveillance phase is raised. In detection-tracking phase, events such as attack are detected through analysis of movement trajectory of an object and it is notified to image processing system.

The system proposed by this study requires at least one camera and image processing equipment that stores and processes database and image data of specific face and tracks trajectory of movement of an object.

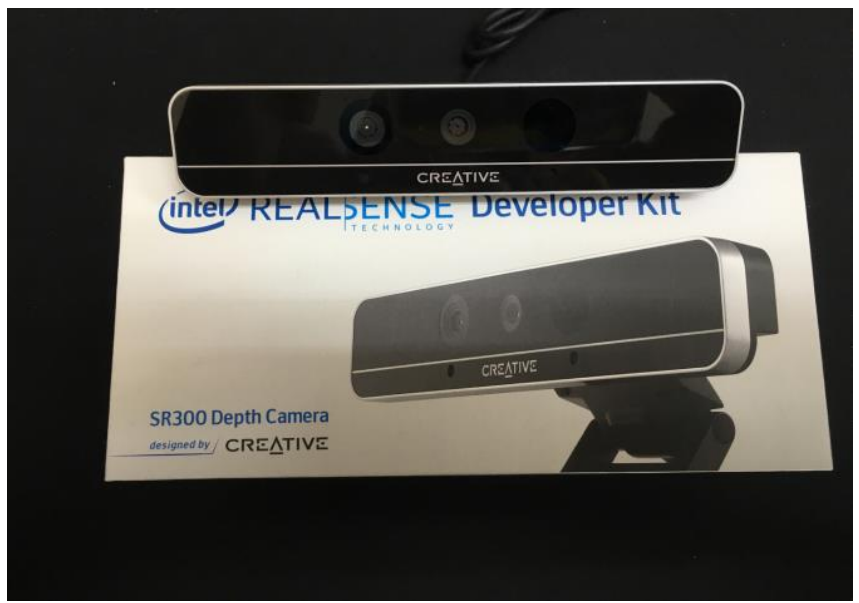

Figure 5. Intel REAL SENSE Developer Kit SR300

Intel Real Sense Development Kit is used as camera that collects inner image as seen in Figure 5. REALSENSE has the specifications as seen in Table 2

Table 2. The Specifications of REAL SENSE

\begin{tabular}{|l|l|}
\hline OS Support & Microsoft Windows 1 10 \\
\hline Connectivity & USB 3.0 \\
\hline Recommended CPU & Intel 6th generation processor or above \\
\hline Video Streaming & $\begin{array}{l}720 \mathrm{p} \text { at } 60 \text { frames per second video capture optimized for streaming } \\
1080 \mathrm{p} \text { at } 30 \text { frames per second video capture for video conferencing }\end{array}$ \\
\hline Depth Range & Approximately $20 \mathrm{~cm}$ to $150 \mathrm{~cm}$ (Software optimized in this range) \\
\hline Microphone & dual-array microphones \\
\hline
\end{tabular}




\begin{tabular}{|l|l|}
\hline SDK Support & $\begin{array}{l}\text { Dynamic background segmentation } \\
\text { 3D scanning } \\
\text { Facial recognition and hand gesture recognition }\end{array}$ \\
\hline
\end{tabular}

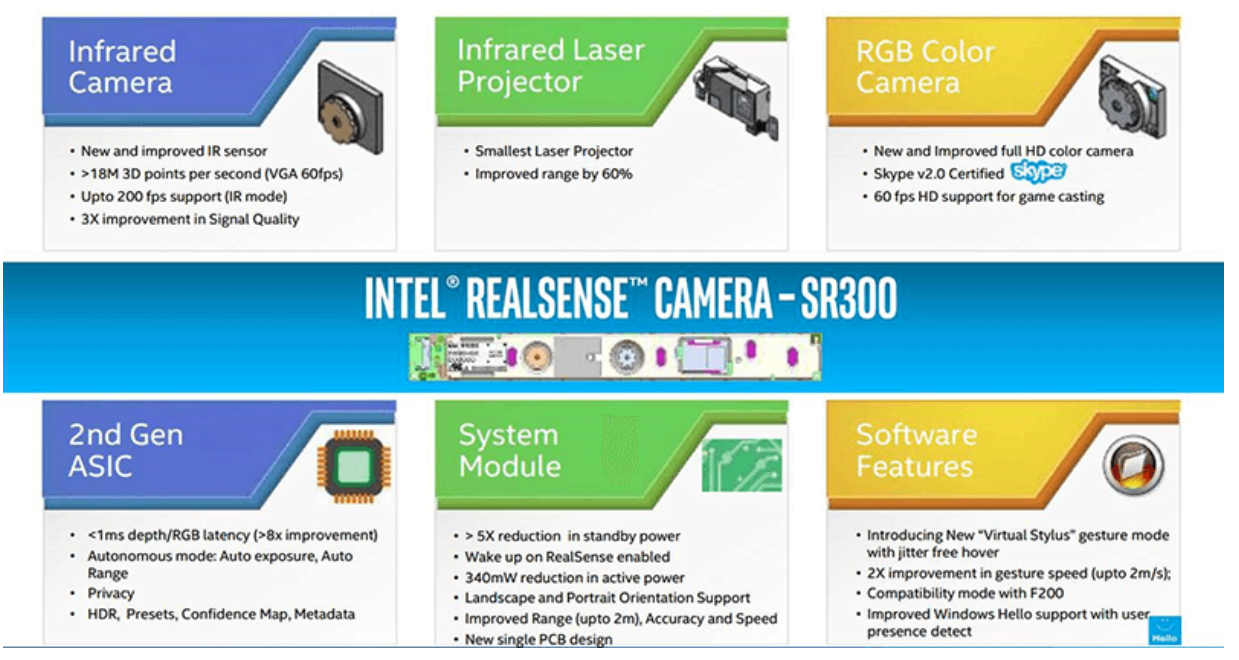

Figure 6. Hardware Specification of REAL SENSE

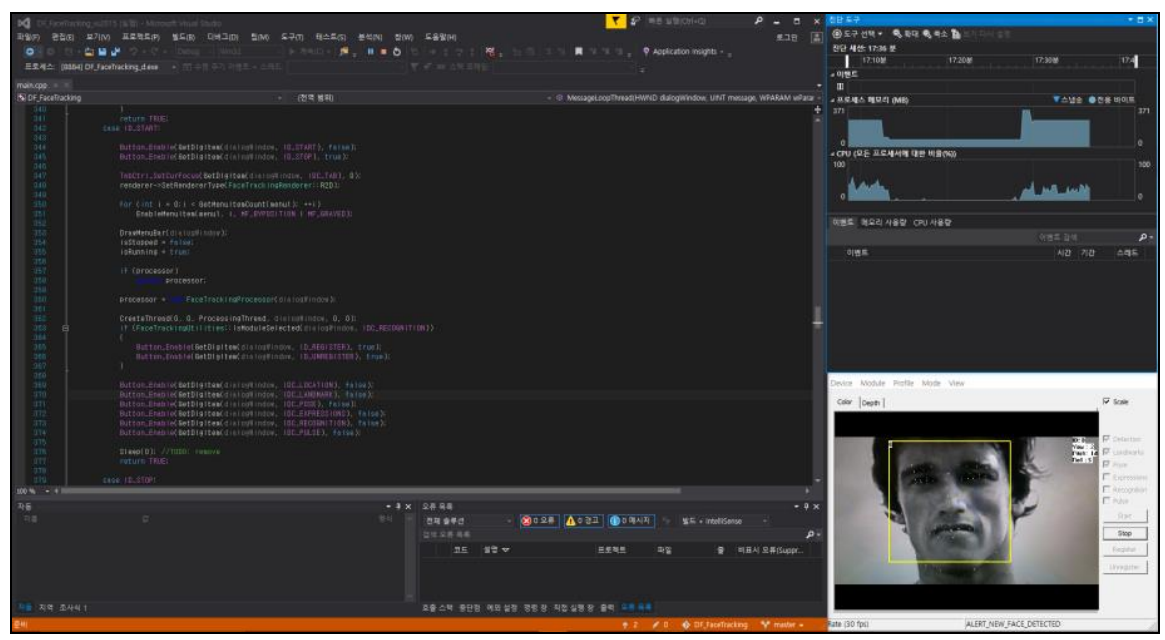

Figure 7. Development Environment

Figure 6 shows a hardware specification of REAL SENSE. It has Infrared Camera, Infrared Laser Projector, RGB Color Camera, $2^{\text {nd }}$ Gen ASIC, System Module and Software. Also, Figure 7 shows a development environment of this study. It is used that Visual Studio 2015 on Window 10. Also REAL SENSE needs the to operate over intel $4^{\text {th }}$ generation CPU chips. 


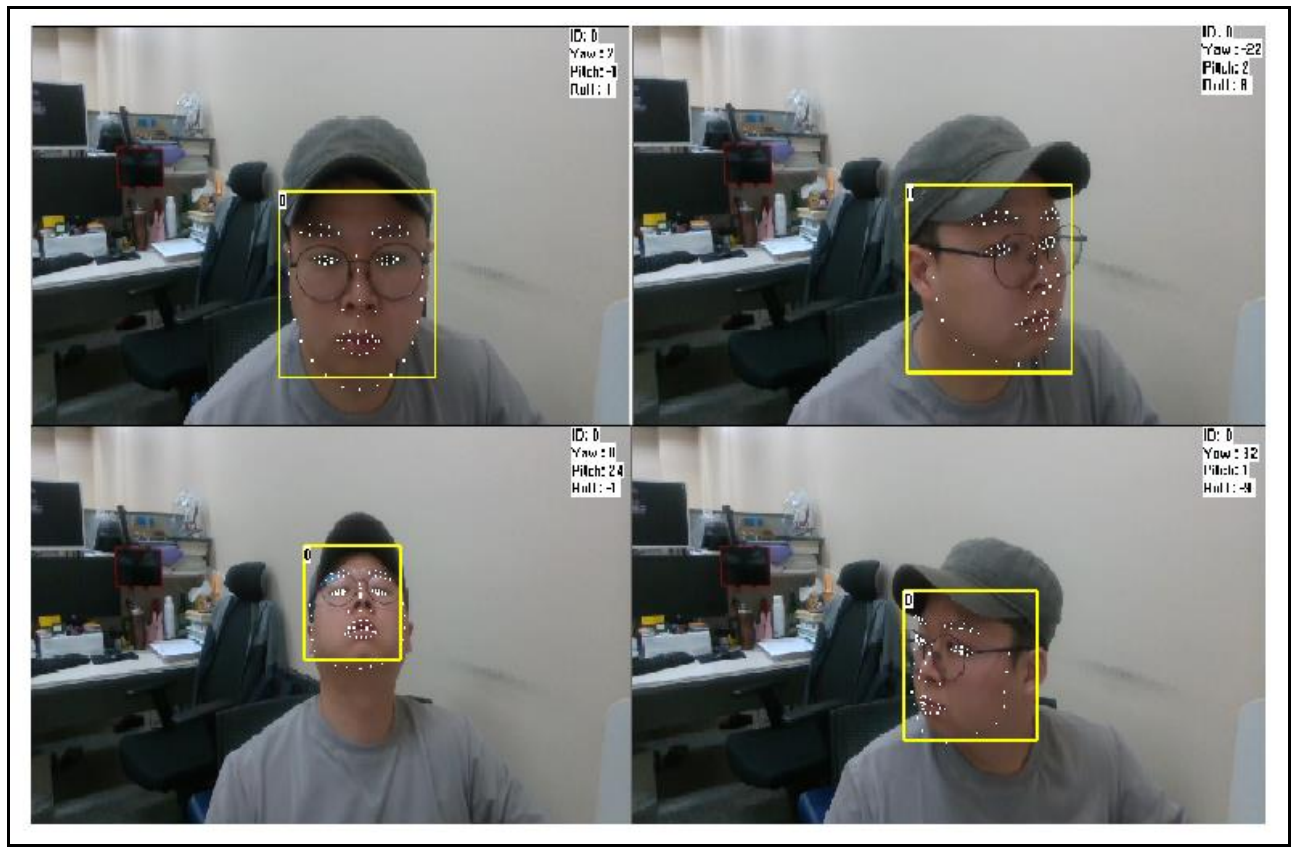

Figure 8. Face Detection

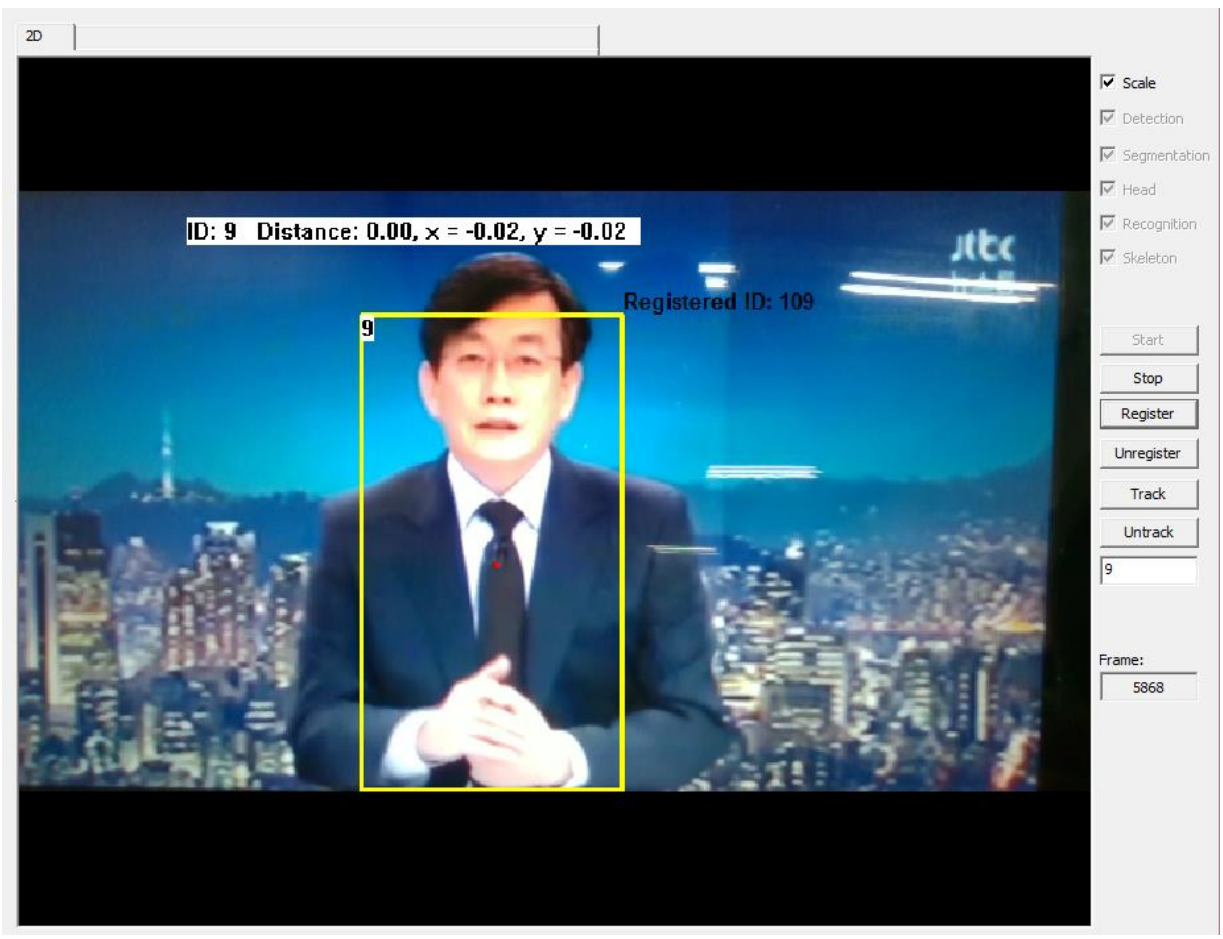

Figure 9. Object(Human) Detection 


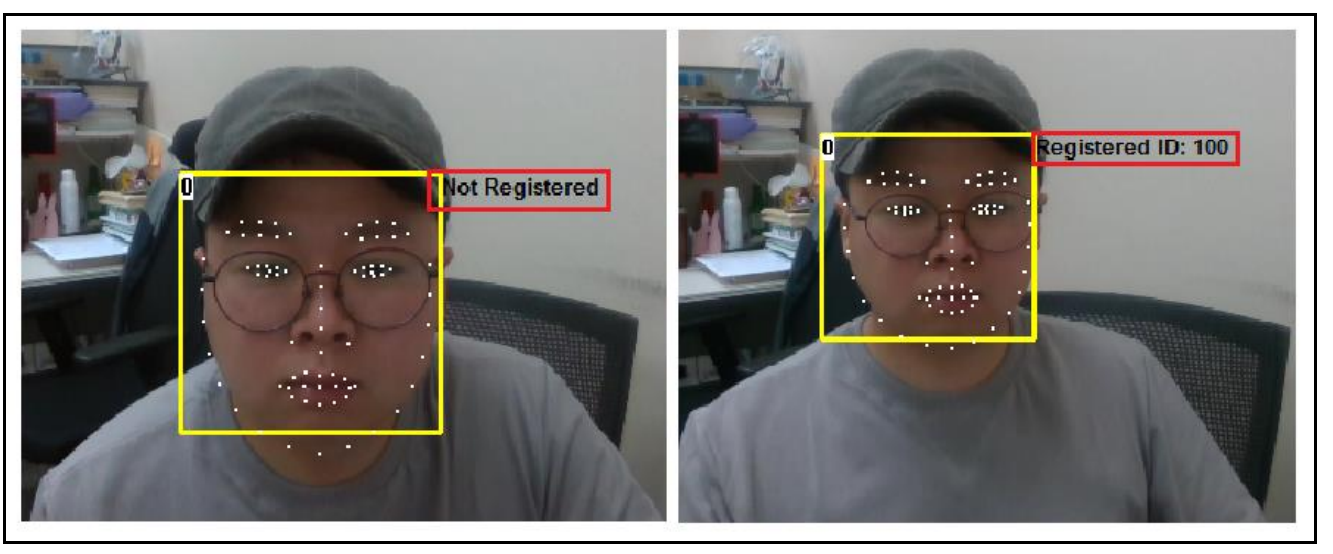

Figure 10. Face Recognition

Figure 8 shows a screen that the face is detected in the proposed system. As you can see, the face is detected irrespective of angle of face reflected in a screen. Figure 10 shows a screen that is a result is a of face recognition that is output by comparing face database and input specific face. On the left is a case that there is no face in face database while on the right is a case that there is face in face database and registration number of specific face is output. Figure 9 shows a screen that human body is detected in proposed system.

\section{Conclusions}

The purpose of this study is to prevent secondary accident that may occur and cope with violence on driver by detecting specific events regarding the taxi driver or bus driver. In this paper, a limited type of experiment was conducted. In other words, the experiment was carried out in a laboratory and not in a taxi or bus. Face detection and recognition was conducted in a smooth manner but event detection through analysis of object movement trajectory varies depending on surrounding environment such as illumination or object overlapping. It is expected that in taxi or bus which has more diverse environment, event detection rate will be lowered. In the next study, the author of this paper will try to raise event detection rate in actual environment such as taxi or bus.

\section{References}

[1] Jae-Gon Yoo, Dong-Kyun Kim, Seung Joo Choi, Handong Lee and Jong-Bae Lee, "A Study on Detecting Driver Assaulting Event Using Intel Real-Sense Camera”, 2016 International Conference on Future Information \& Communication Engineering (ICFICE), 23th-25 ${ }^{\text {th }}$ June, 2016, Novotel Danang Premier Han River, Danang, Vietnam, Vol.8, No.1, 308-309

[2] IDEC News Letter Technology Trends, Han-Dong Il, "Real-time face detection technology research trends", p08-p13, 2010.12

[3] A. Yilmaz, O. Javed and M. Shah, "Object Tracking: A Survey,” ACM Computing Surveys, vol. 38, no.4, Article 13, 2006.

[4] B. Wu, X. Song, V. Kumar Singh and R. Nevatia, "Evaluation of USC Human Tracking System for Surveillance Videos," LectureNotes in Computer Science, vol.4625, pp.191-196, 2008

[5] YANG.MH, KRIEGMAN.D, and AHUJA.N,"Detecting faces in images: A survey”, IEEE Trans, Patt, Anal,Intell, 24, PP 34?58, Mach, 2002

[6] Signal Processing Institute, Swiss Federal Institute of Technology http://scgwww.epfl.ch/

[7] Biometric Systems Lab, University of Bologna http://bias.csr.unibo.it/research/biolab/, "Face Recognition CPSC 601 Biometric Course. Topics Challenges in face recognition Face detection Face recognition Advantages and disadvantages."

[8] SK Planet Technology Blog, Image Processing Team, Un-Dong Jang, "Introduction of Face Recognition", http://readme.skplanet.com/?p=4308, 2013.05.08

[9] Telecommunications Technology Association (TTA), "Technical Analysis Report (Object Tracking and Trajectory of the Video Surveillance System)”, TTA Technical Report, 2009. 


\section{Authors}
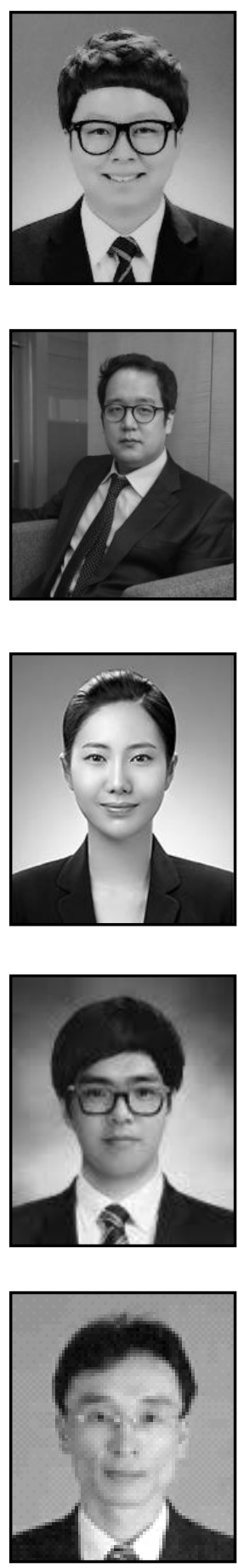

Jae-Gon Yoo, he received his bachelor's degree of Computer Science in Soongsil University, Seoul(2015). And he is studying his master's degree of software engineering in the Graduate School of Software, Soongsil University, Seoul. His current research interests include Open Source Software and Software Engineering.

Dong-Kyun Kim, he received his bachelor's degree of Business Administration in Hanyang University,Seoul (2001). He is studying his doctor's degree of Information Technology Policy \& Management in Graduated Soongsil University, Seoul. His current research interests include Open Source Software and Management of Technology.

Seung Joo Choi, she received her bachelor's degree of Multimedia Science in Sookmyung Women's University, Seoul(2016). Now she is studying in the Graduate School of Software, Soongsil University, Seoul, Korea. Her research interests focus on Mobile Application, Web Application, and Open Source Software.

Handong Lee, he received his bachelor's degree of Science in Computer Engineering in Korea University of Technology and Education(2015). Now he is in the Graduate School of Software, Soongsil University, Seoul, Korea for master degree. His research interests focus on Software Engineering, and Open Source Software.

Jong-Bae Kim, he received his bachelor's degree of Business Administration in University of Seoul, Seoul(1995) and master's degree(2002), doctor's degree of Computer Science in Soongsil University, Seoul(2006). Now he is a professor in the Graduate School of Software, Soongsil University, Seoul, Korea. His research interests focus on Software Engineering, and Open Source Software. 
International Journal of Control and Automation

Vol. 10, No. 2 (2017) 